\title{
Reduced Vertex Set Result for Interval Semidefinite Optimization Problems
}

\author{
G. Calafiore - F. Dabbene
}

Published online: 26 April 2008

(C) Springer Science+Business Media, LLC 2008

\begin{abstract}
In this paper we propose a reduced vertex result for the robust solution of uncertain semidefinite optimization problems subject to interval uncertainty. If the number of decision variables is $m$ and the size of the coefficient matrices in the linear matrix inequality constraints is $n \times n$, a direct vertex approach would require satisfaction of $2^{n(m+1)(n+1) / 2}$ vertex constraints: a huge number, even for small values of $n$ and $m$. The conditions derived here are instead based on the introduction of $m$ slack variables and a subset of vertex coefficient matrices of cardinality $2^{n-1}$, thus reducing the problem to a practically manageable size, at least for small $n$. A similar size reduction is also obtained for a class of problems with affinely dependent interval uncertainty.
\end{abstract}

Keywords Semidefinite optimization · Robustness · Linear matrix inequalities · Uncertainty

\section{Introduction}

Semidefinite convex optimization problems (SDPs) deal with the minimization of a linear objective subject to a set of linear matrix inequality (LMI) constraints on the

Communicated by B.T. Polyak.

This work is supported by MIUR under the FIRB project "Learning, Randomization and Guaranteed Predictive Inference for Complex Uncertain Systems," and by CNR RSTL funds.

G. Calafiore ( $₫)$

Dipartimento di Automatica e Informatica, Politecnico di Torino, Torino, Italy

e-mail: giuseppe.calafiore@polito.it

F. Dabbene

IEIIT-CNR, Politecnico di Torino, Torino, Italy

e-mail: fabrizio.dabbene@polito.it 
design variable $x \in \mathbb{R}^{m}$ and take the standard form

$$
\begin{array}{ll}
\min & c^{\top} x, \\
\text { s.t. } & F(x)=F_{0}+\sum_{k=1}^{m} x_{k} F_{k} \preceq 0,
\end{array}
$$

where $F_{k}, k=0, \ldots, m$, are symmetric matrices; see, e.g., [1]. Efficient polynomialtime solution techniques exist for this class of problems, such as those based on primal-dual interior-point methods, see [2-4].

In the last years, the consideration that most real-world problems unavoidably entail a certain degree of uncertainty stimulated the research on robust solutions to uncertain SDP problems, see, e.g., [5, 6]. In this setting, the problem data $\left(F_{0}, \ldots, F_{k}\right)$ are assumed to be affected by bounded uncertainty, and a solution is said to be robust if it is guaranteed to satisfy the constraints for all admissible uncertainty values.

Unfortunately, tractable necessary and sufficient conditions for the solution of robust SDP problems are available only for very special problem classes, while the general situation is known to be NP-hard, see for instance [5-8]. Various relaxation approaches have hence been proposed to conservatively solve these problems. In particular, in [6] the authors provide upper bounds on the optimal solution (i.e. the objective is minimized subject to sufficient conditions for robust satisfaction of the uncertain LMIs) for the case when the uncertainty enters the data in a linear fractional form, while in [9] the case of polytopic uncertainty is considered and a numerically tractable relaxation of the problem is provided, together with an a-priori bound on the degree of conservativeness of the approximation.

In this paper, we consider the case when the LMI coefficient matrices $F_{k}, k=$ $0,1, \ldots, m$, are symmetric interval matrices, that is, symmetric matrices whose entries are bounded independently in given intervals. It is a well-known fact, easily proven by convexity arguments, that in this case the uncertain LMI condition is robustly satisfied whenever it is satisfied for all the $2^{n(m+1)(n+1) / 2}$ vertex matrices, that is the matrices obtained by setting each matrix entry to its upper or lower limit, see Sect. 2.1. However, recent results in the literature (see [10,11] and the references therein) suggest that the number of vertices can actually be reduced in certain special classes of interval optimization problems. Motivated by these ideas, we develop in Section 2 an equivalent formulation of a robust interval SDP which is based on the introduction of $m$ slack variables and requires satisfaction of only $2^{n-1}$ specially selected vertex matrices. Although this result is still exponential in the matrix dimension $n$, the number of required vertex constraints can be manageable by currently available solvers, for reasonable values of $n$. Exponential growth of the number of vertices is not surprising and cannot be avoided in general (unless $\mathrm{P}=\mathrm{NP}$ ), since it was already proven in [12] that even the simpler problem of checking robust positive semidefiniteness of a symmetric interval matrix is NP-hard. When even this reduced number of vertices would lead to a problem of unmanageable size, we show in Sect. 2.3 that a specific SDP relaxation can be introduced, so that an upper bound on the objective can be computed in polynomial time. As a by-product of our reduced vertex set result, we also obtain that for a very specific class of uncertain SDPs, namely linear programs (LP) affected by interval uncertainty, the robust optimization problem can be recast exactly as a standard linear program with slack variables and hence solved in polynomial time. 
Finally, in Sect. 3 we present a result that holds when the uncertainty affecting the data is not completely independent, but it is instead represented by a linear transformation of a $p \times q$ interval matrix. In this case, a weaker result holds, which prescribes to impose satisfaction of the LMI constraints at $2^{p+q-1}$ selected vertices.

\subsection{Related Literature}

The results of this paper are related to a classical work of Rohn [11] on interval symmetric matrices and to a recent paper of Alamo et al. [10], which provides a new and interesting vertex set result for a class of problems arising in a robust control setting. In particular, our interest in the subject and the developments in this paper have been directly inspired by the results in [10].

In details, in the work [11] a reduced vertex set result is provided for checking negative-definiteness of a symmetric interval matrix. Specifically, Theorem 2 of [11] states that a symmetric interval matrix of dimension $n \times n$ is robustly negative semidefinite if and only if $2^{n-1}$ special vertex matrices are negative semidefinite. Notice that the result of [11] is an analysis result, related to the problem of checking if a property (negative semidefiniteness) holds robustly. Instead, the problem considered in this paper is a design one, that is, the goal is to find a design vector $x$ such that an interval linear matrix inequality in $x$ is robustly satisfied. It is thus interesting to notice that the same vertex growth factor applies to both robustness analysis and design problems. The result of [11] is recovered in our framework as a special case for $m=0$ (no design variables).

The developments in Sect. 3 deal instead with a class of uncertain SDP problems affected by affinely transformed interval uncertainty. Section 3 is closely related to the work in [10]. In particular, in Remark 3.1 we show that, under the proposed setting, the main vertex cardinality result in [10] can be re-derived and improved by an halving factor.

\subsection{Notations}

For a vector $x$, the $i$ th element is denoted by $x_{i}$. The element in the $i$ th row and $j$ th column of a matrix $X$ is denoted either by $[X]_{i j}$, or by $X_{i j}$. For $X \in \mathbb{R}^{n, m}$, the notation $X \leq 0$ (resp. $X<0$ ) denotes elementwise nonstrict (resp. strict) inequality. The notation $|X|$ denotes a matrix whose $(i, j)$ th element is $\left|X_{i j}\right| . \mathbb{S}^{n}$ denotes the subspace of symmetric $n \times n$ real matrices. For $X \in \mathbb{S}^{n}$, the notation $X \preceq 0$ (resp. $X \prec$ $0)$ specifies that $X$ is negative semidefinite (resp. negative definite). If $X_{1}, \ldots, X_{k}$ are matrices, the notation $\operatorname{diag}\left(X_{1}, \ldots, X_{k}\right)$ denotes a block-diagonal matrix having $X_{1}, \ldots, X_{k}$ as diagonal blocks. If $x \in \mathbb{R}^{n}$, the notation $\operatorname{diag}(x)$ denotes a diagonal matrix with the elements of $x$ on the diagonal. The operator $\odot$ denotes the Hadamard (entrywise) matrix product. The set of diagonal matrices of signs is defined as

$$
\mathcal{S}^{n} \doteq\left\{\operatorname{diag}\left(s_{1}, \ldots, s_{n}\right): s_{i}= \pm 1, i=1, \ldots, n\right\} .
$$

The cardinality of this set is $\operatorname{card}\left(\mathcal{S}^{n}\right)=2^{n}$. 


\section{Interval SDPs}

Consider an uncertain linear matrix inequality restriction on the variables $x_{1}, \ldots, x_{m}$,

$$
F(x)=F_{0}+\sum_{k=1}^{m} x_{k} F_{k} \preceq 0,
$$

where $F_{k} \in \mathbb{S}^{n}, k=0,1, \ldots, m$, are symmetric interval coefficient matrices. Namely, we assume that

$$
F_{k}=F_{k}\left(\Delta_{k}\right)=\bar{F}_{k}+\Delta_{k}, \quad k=0,1, \ldots, m,
$$

where $\bar{F}_{k} \in \mathbb{S}^{n}$ are given and $\Delta_{k}$ are only known to belong to the interval sets

$$
\mathcal{D}_{k} \doteq\left\{\Delta \in \mathbb{S}^{n}:|\Delta| \leq B_{k}\right\}
$$

where $B_{k} \geq 0$ is a symmetric matrix containing the bounds on the entries of $\Delta$.

A robust interval LMI is then defined as the following semi-infinite convex constraint

$$
F_{0}\left(\Delta_{0}\right)+\sum_{k=1}^{m} x_{k} F_{k}\left(\Delta_{k}\right) \preceq 0, \quad \forall \Delta_{k} \in \mathcal{D}_{k}, k=0,1, \ldots, m .
$$

In this paper, we treat the two robustness problems defined next.

Problem 2.1 (Robust Feasibility of Interval LMI) Given $x \in \mathbb{R}^{m}$, check if (2) holds.

Problem 2.2 (Robust Solution of Interval SDP) Given $c \in \mathbb{R}^{m}$, find $x \in \mathbb{R}^{m}$ such that $c^{\top} x$ is minimized subject to the constraints (2).

These interval problems found many applications in different fields, ranging from numerical analysis and robust linear algebra to engineering design. As a simple motivating example, we next illustrate a problem arising in the context of topology optimization and vibration control of discrete (or discretized) mechanical structures.

Example 2.1 (Truss Topology Optimization) A classical problem in structural design is to determine the cross-sectional areas $x_{i}$ of a truss structure so to minimize the total weight of the structure while guaranteeing a lower bound on the structure fundamental modal frequency. Formally, for a desired frequency $\Omega \geq 0$, one has to solve an optimization problem of the form (see, e.g., $[13,14]$ )

$$
\begin{array}{ll}
\min _{x} & V(x), \\
\text { s.t. } & x_{\mathrm{lb}} \leq x \leq x_{\mathrm{up}}, \\
& M(x) \Omega^{2}-K(x) \preceq 0,
\end{array}
$$

where the weight of the structure $V(x)$ is a linear function of the design variable $x$, the matrices $K(x)$ and $M(x)$ represent respectively the stiffness and the mass matrix 
of the structure, and constraint (3c) specifies that the fundamental modal frequency should be higher than $\Omega$. The vectors $x_{\mathrm{lb}}, x_{\mathrm{ub}}$ contain lower and upper bounds on the cross-sectional areas, respectively. The mass and stiffness matrices are affine functions of $x$, that is,

$$
K(x)=K_{0}+\sum_{k=1}^{m} x_{k} K_{k}, \quad M(x)=M_{0}+\sum_{k=1}^{m} x_{k} M_{k},
$$

where $M_{k}, K_{k}, k=0, \ldots, m$, are symmetric matrices. Since these matrices depend on geometric and material characteristics of the structure, it is natural to assume an interval uncertainty over them, in which case

$$
\begin{aligned}
& K_{k}=\bar{K}_{k}+\Delta_{k}^{K}, \quad k=0, \ldots, m, \\
& M_{k}=\bar{M}_{k}+\Delta_{k}^{M}, \quad k=0, \ldots, m .
\end{aligned}
$$

In this situation, the designer may be interested in determining a design that works best in the worst-case scenario, that is in considering a robust version of problem (3). It can be easily seen that the robust version of the constraint $(3 \mathrm{c})$ is readily rewritten as an interval LMI of the form (2) by letting

$$
\bar{F}_{k}=\bar{M}_{k} \Omega^{2}-\bar{K}_{k}, \quad \Delta_{k}=\Delta_{k}^{M} \Omega^{2}-\Delta_{k}^{K}, \quad k=0, \ldots, m .
$$

\subsection{Naive Vertex Solution Approach}

We remark that there exist a straightforward (but computationally inefficient) way to solve Problems 2.1 and 2.2. Indeed, it can be seen easily that every matrix in the set $\mathcal{D}_{k}$ can be written as a convex combination of vertex matrices belonging to the set

$$
\mathcal{D}_{k}^{v} \doteq\left\{\Delta \in \mathbb{S}^{n}:\left|\Delta_{i j}\right|=\left[B_{k}\right]_{i j}, 1 \leq i \leq j \leq n\right\}
$$

which has cardinality $2^{n(n+1) / 2}$. Then, the following lemma can be proved by elementary convexity arguments.

Lemma 2.1 The semi-infinite LMI constraint (2) is equivalent to the following finite set of vertex LMI contraints

$$
F_{0}\left(\Delta_{0}\right)+\sum_{k=1}^{m} x_{k} F_{k}\left(\Delta_{k}\right) \preceq 0, \quad \forall \Delta_{k} \in \mathcal{D}_{k}^{v}, k=0,1, \ldots, m .
$$

\section{Problem 2.2 is thus equivalent to the SDP}

$$
\begin{array}{ll}
\min & c^{\top} x, \\
\text { s.t. } & (4) .
\end{array}
$$

In the latter minimization problem, the infinite number of constraints of Problem 2.2 has been replaced by a finite number of vertex constraints. Notice however that this 
number can be very large already for very small $n$ and $m$. For instance, for $n=$ $m=3$ the number of constraints is $16,777,216$, and it becomes $1.1259 \times 10^{15}$ for $n=m=4$. Hence, Lemma 2.1 has a theoretical interest, but it can rarely be applied in practice. In the next section we derive a new result that shows how the number of vertices can be drastically reduced and made independent of $m$, thus leading to a more manageable solution approach.

\subsection{A Result with Reduced Vertex Set}

We first establish the following preliminary lemma, which is instrumental for proving the main result.

Lemma 2.2 Given $x \in \mathbb{R}^{m}$, the robustness condition (2) is satisfied if and only if

$$
v^{\top} \bar{F}(x) v+|v|^{\top} B(|x|)|v| \leq 0, \quad \forall v \in \mathbb{R}^{n},
$$

where we define

$$
\begin{aligned}
& \bar{F}(x) \doteq \bar{F}_{0}+\sum_{k=1}^{m} x_{k} \bar{F}_{k}, \\
& B(|x|) \doteq B_{0}+\sum_{k=1}^{m}\left|x_{k}\right| B_{k} .
\end{aligned}
$$

Proof The proof of this result follows a reasoning similar to the one introduced in [10] and [11]. The robust interval LMI (2) is satisfied if and only if

$$
v^{\top} F_{0}\left(\Delta_{0}\right) v+\sum_{k=1}^{m} x_{k} v^{\top} F_{k}\left(\Delta_{k}\right) v \leq 0
$$

holds for all $\Delta_{k} \in \mathcal{D}_{k}$ and all $v \in \mathbb{R}^{n}$, that is, if and only if

$$
\max _{\Delta_{0} \in \mathcal{D}_{0}} v^{\top} F_{0}\left(\Delta_{0}\right) v+\sum_{k=1}^{m} \max _{\Delta_{k} \in \mathcal{D}_{k}} x_{k} v^{\top} F_{k}\left(\Delta_{k}\right) v \leq 0, \quad \forall v \in \mathbb{R}^{n} .
$$

Notice that

$$
\begin{aligned}
& \max _{\Delta_{0} \in \mathcal{D}_{0}} v^{\top} F_{0}\left(\Delta_{0}\right) v+\sum_{k=1}^{m} \max _{\Delta_{k} \in \mathcal{D}_{k}} x_{k} v^{\top} F_{k}\left(\Delta_{k}\right) v \\
& =v^{\top} \bar{F}_{0} v+\sum_{k=1}^{m} x_{k} v^{\top} \bar{F}_{k} v+\max _{\Delta_{0} \in \mathcal{D}_{0}} v^{\top} \Delta_{0} v+\sum_{k=1}^{m} \max _{\Delta_{k} \in \mathcal{D}_{k}} x_{k} v^{\top} \Delta_{k} v \\
& =v^{\top} \bar{F}(x) v+\max _{\Delta_{0} \in \mathcal{D}_{0}} v^{\top} \Delta_{0} v+\sum_{k=1}^{m} \max _{\Delta_{k} \in \mathcal{D}_{k}} x_{k} v^{\top} \Delta_{k} v .
\end{aligned}
$$


Considering the second term in the previous summation, we have that

$$
\max _{\Delta_{0} \in \mathcal{D}_{0}} v^{\top} \Delta_{0} v=\max _{\Delta_{0} \in \mathcal{D}_{0}}\left(\sum_{i=1}^{n} v_{i}^{2}\left[\Delta_{0}\right]_{i i}+2 \sum_{1 \leq i \leq j \leq n} v_{i} v_{j}\left[\Delta_{0}\right]_{i j}\right) .
$$

The maximum in this expression is attained by choosing

$$
\left[\Delta_{0}\right]_{i i}=\left[B_{0}\right]_{i i} \text { and }\left[\Delta_{0}\right]_{i j}=\operatorname{sign}\left(v_{i} v_{j}\right)\left[B_{0}\right]_{i j} \text {, }
$$

which yields

$$
\max _{\Delta_{0} \in \mathcal{D}_{0}} v^{\top} \Delta_{0} v=\sum_{i=1}^{n} v_{i}^{2}\left[B_{0}\right]_{i i}+2 \sum_{1 \leq i \leq j \leq n}\left|v_{i} v_{j}\right|\left[B_{0}\right]_{i j}=|v|^{\top} B_{0}|v| .
$$

Similarly, considering the third term in (7), we have

$$
\max _{\Delta_{k} \in \mathcal{D}_{k}} x_{k} v^{\top} \Delta_{k} v=\max _{\Delta_{k} \in \mathcal{D}_{k}}\left(\sum_{i=1}^{n} x_{k} v_{i}^{2}\left[\Delta_{k}\right]_{i i}+2 \sum_{1 \leq i \leq j \leq n} x_{k} v_{i} v_{j}\left[\Delta_{k}\right]_{i j}\right) .
$$

For given $x_{k}$, the maximum in this expression is attained by choosing

$$
\left[\Delta_{k}\right]_{i i}=\operatorname{sign}\left(x_{k}\right)\left[B_{k}\right]_{i i} \quad \text { and } \quad\left[\Delta_{k}\right]_{i j}=\operatorname{sign}\left(x_{k} v_{i} v_{j}\right)\left[B_{k}\right]_{i j},
$$

yielding

$$
\max _{\Delta_{k} \in \mathcal{D}_{k}} x_{k} v^{\top} \Delta_{k} v=\sum_{i=1}^{n}\left|x_{k}\right| v_{i}^{2}\left[B_{k}\right]_{i i}+2 \sum_{1 \leq i \leq j \leq n}\left|x_{k}\right|\left|v_{i} v_{j}\right|\left[B_{k}\right]_{i j}=\left|x_{k}\right||v|^{\top} B_{k}|v|,
$$

thus concluding the proof.

We are now in position to state the following corollary, which provides a reduced vertex set solution for Problem 2.1.

Corollary 2.1 (Robust Feasibility with Reduced Vertex Set) Given $x \in \mathbb{R}^{m}$, the semiinfinite condition (2) is satisfied if and only if

$$
\bar{F}(x)+S B(|x|) S \preceq 0, \quad S=\operatorname{diag}(1, \tilde{S}), \forall \tilde{S} \in \mathcal{S}^{n-1},
$$

where $\bar{F}(x)$ and $B(|x|)$ are defined in (5), (6) and $\mathcal{S}^{n-1}$ is the set of $(n-1) \times(n-1)$ diagonal matrices of signs. Condition (8) consists of a finite number $2^{n-1}$ of vertex conditions.

Proof Suppose first that (2) holds, that is,

$$
\bar{F}(x)+\Delta_{0}+\sum_{k=1}^{m} x_{k} \Delta_{k} \preceq 0, \quad \forall \Delta_{k} \in \mathcal{D}_{k}, k=0,1, \ldots, m .
$$


Then in particular, for any $\tilde{S} \in \mathcal{S}^{n-1}$, let $S=\operatorname{diag}(1, \tilde{S})$ and choose

$$
\begin{aligned}
& \bar{\Delta}_{0}=S B_{0} S \in \mathcal{D}_{0}, \\
& \bar{\Delta}_{k}=\operatorname{sign}\left(x_{k}\right) S B_{k} S \in \mathcal{D}_{k}, \quad k=1, \ldots, m .
\end{aligned}
$$

Then, it must hold that

$$
\bar{F}(x)+S B_{0} S+\sum_{k=1}^{m}\left|x_{k}\right| S B_{k} S \preceq 0,
$$

which proves the first implication.

Conversely, suppose that (8) holds, and notice that $S B(|x|) S=(-S) B(|x|)(-S)$. This implies that the condition in (8) actually holds for all $S \in \mathcal{S}^{n}$. Therefore, for all $v \in \mathbb{R}^{n}$, it holds that

$$
v^{\top} \bar{F}(x) v+v^{\top} S B(|x|) S v \leq 0, \quad \forall S \in \mathcal{S}^{n}
$$

Hence, choosing $S=\operatorname{diag}\left(\operatorname{sign}\left(v_{1}\right), \ldots, \operatorname{sign}\left(v_{n}\right)\right)$, it must hold that

$$
v^{\top} \bar{F}(x) v+|v|^{\top} B(|x|)|v| \leq 0, \quad \forall v \in \mathbb{R}^{n} .
$$

By Lemma 2.2, this latter condition implies satisfaction of (2), thus concluding the proof.

The result given in Corollary 2.1 is useful for robustness analysis (Problem 2.1). Notice that in the special case of $m=0$ (no design variables) the vertex matrices in (8) coincide with those required in Theorem 2 of [11] for checking negativesemidefiniteness of symmetric interval matrices.

We next state the main result of the paper, which provides a reduced vertex set condition for the solution of the robust design problem (Problem 2.2).

Theorem 2.1 (Robust Optimization with Reduced Vertex Set) The robust interval $S D P$ problem (Problem 2.2) is equivalent to the following SDP in the variables $x, \xi \in$ $\mathbb{R}^{m}$

$$
\begin{array}{ll}
\min & c^{\top} x, \\
\text { s.t. } & \bar{F}(x)+S B(\xi) S \preceq 0, \quad S=\operatorname{diag}(1, \tilde{S}), \forall \tilde{S} \in \mathcal{S}^{n-1}, \\
& x_{k} \leq \xi_{k}, \quad k=1, \ldots, m, \\
& -x_{k} \leq \xi_{k}, \quad k=1, \ldots, m,
\end{array}
$$

where

$$
B(\xi)=B_{0}+\sum_{k=1}^{m} \xi_{k} B_{k}
$$


Proof We show that the robust LMI conditions (2) and (9b)-(9d) are equivalent. Suppose first that (2) holds for some $x \in \mathbb{R}^{m}$. Then, (8) holds by Corollary 2.1, hence (9b)-(9d) hold by taking $\xi_{k}=\left|x_{k}\right|$.

Conversely, suppose that (9b)-(9d) hold, and notice that (9c), (9d) imply $\left|x_{k}\right| \leq \xi_{k}$. Since $S B(\xi) S=(-S) B(\xi)(-S)$, condition (9b) implies that $\bar{F}(x)+S B(\xi) S \preceq 0$ for all $S \in \mathcal{S}^{n}$. Hence, for all $S \in \mathcal{S}^{n}$,

$$
v^{\top} \bar{F}(x) v+v^{\top} S B_{0} S v+\sum_{k=1}^{m} \xi_{k} v^{\top} S B_{k} S v \leq 0, \quad \forall v \in \mathbb{R}^{n} .
$$

In particular, for $S=\operatorname{diag}\left(\operatorname{sign}\left(v_{1}\right), \ldots, \operatorname{sign}\left(v_{n}\right)\right)$, we have

$$
v^{\top} \bar{F}(x) v+|v|^{\top} B_{0}|v|+\sum_{k=1}^{m} \xi_{k}|v|^{\top} B_{k}|v| \leq 0, \quad \forall v \in \mathbb{R}^{n} .
$$

Now, since $B_{k} \geq 0$, then $|v|^{\top} B_{k}|v| \geq 0$. Therefore $\left|x_{k}\right| \leq \xi_{k}$ implies that $\xi_{k}|v|^{\top} B_{k}|v| \geq\left|x_{k}\right||v|^{\top} B_{k}|v|$; thus, for all $v \in \mathbb{R}^{n}$,

$$
\begin{aligned}
& v^{\top} \bar{F}(x) v+|v|^{\top} B_{0}|v|+\sum_{k=1}^{m}\left|x_{k}\right||v|^{\top} B_{k}|v| \\
& \leq v^{\top} \bar{F}(x) v+|v|^{\top} B_{0}|v|+\sum_{k=1}^{m} \xi_{k}|v|^{\top} B_{k}|v| \leq 0 .
\end{aligned}
$$

This implies satisfaction of (2), by Lemma 2.2, which concludes the proof.

Remark 2.1 (Vertex Complexity) Theorem 2.1 shows that the infinite set of constraints in Problem 2.2 can be substituted by an equivalent finite set of $2^{n-1}$ vertex LMIs. The number of vertices is thus independent of $m$ and it is drastically reduced with respect to the case considered in Sect. 2.1.

Looking more closely to condition (9b) in Theorem 2.1, we see that when $B(\xi)$ and $S$ commute (i.e. when $B(\xi)$ is diagonal) then, since $S S=I$, (9b) reduces to the single LMI constraint $\bar{F}(x)+B(\xi) \preceq 0$. This situation happens for instance in the special case of interval linear programs, which is illustrated in the next example.

Example 2.2 (Special Case: Interval Linear Programs) Consider a standard linear programming problem (LP),

$$
\begin{array}{ll}
\min & c^{\top} x, \\
\text { s.t. } & A x-b \leq 0,
\end{array}
$$

with $A \in \mathbb{R}^{n, m}$ and $b \in \mathbb{R}^{n}$. It is straightforward to verify that the linear constraints in this problem can be cast in an equivalent LMI format (1) by taking

$$
F_{0}=\operatorname{diag}(-b), \quad F_{k}=\operatorname{diag}\left(a_{k}\right), \quad k=1, \ldots, m,
$$


where $a_{k}$ denotes the $k$ th column of $A$. Now, if the entries of $A$ and $b$ are assumed to lie in independent intervals, we may write

$$
A=A(\Delta)=\bar{A}+\Delta \odot R, \quad b=b(\delta)=\bar{b}+\delta \odot d,
$$

where $\bar{A} \in \mathbb{R}^{n, m}, \bar{b} \in \mathbb{R}^{n}$ are the nominal matrices, $R \in \mathbb{R}^{n, m}, d \in \mathbb{R}^{n}$ are given positive matrices containing the interval limits, and $\Delta \in \mathbb{R}^{n, m}, \delta \in \mathbb{R}^{n}$ are the uncertainties, which are subject to $|\Delta| \leq 1,|\delta| \leq 1$. The robust interval LP problem

$$
\begin{array}{ll}
\min & c^{\top} x, \\
\text { s.t. } & A(\Delta) x-b(\delta) \leq 0, \quad \forall \Delta, \delta:|\Delta| \leq 1,|\delta| \leq 1,
\end{array}
$$

can therefore be equivalently rewritten in the format of a robust interval SDP, with diagonal nominal coefficient matrices $\bar{F}_{0}=\operatorname{diag}(-\bar{b}), \bar{F}_{k}=\operatorname{diag}\left(\bar{a}_{k}\right)$ and diagonal bound matrices $B_{0}=\operatorname{diag}(d), B_{k}=\operatorname{diag}\left(r_{k}\right), k=1, \ldots, m$, where $\bar{a}_{k}, r_{k}$ are the $k$ th column of $\bar{A}$ and of $R$, respectively.

Let us now apply Theorem 2.1 to this interval SDP. Since $S \in \mathcal{S}^{n}$ and $B_{i}$ are diagonal and $S S=I$, the $S$ terms disappear in equation (9b). It follows that problem (11b) is equivalent to

$$
\begin{array}{ll}
\min & c^{\top} x, \\
\text { s.t. } & \bar{F}_{0}+\sum_{k=1}^{m} x_{k} \bar{F}_{k}+B(\xi) \preceq 0, \\
& x_{k} \leq \xi_{k}, \quad k=1, \ldots, m, \\
& -x_{k} \leq \xi_{k}, \quad k=1, \ldots, m,
\end{array}
$$

where $\xi^{\top}=\left[\xi_{1} \cdots \xi_{m}\right]$ is a vector of slack variables. Converting this diagonally structured SDP back to standard LP format, we finally obtain that (11b) is equivalent to the following standard linear program in the variables $x$ and $\xi$ :

$$
\begin{array}{ll}
\min & c^{\top} x, \\
\text { s.t. } & \bar{A} x-b+R \xi+d \leq 0, \\
& x_{k} \leq \xi_{k}, \quad k=1, \ldots, m, \\
& -x_{k} \leq \xi_{k}, \quad k=1, \ldots, m .
\end{array}
$$

This result, which could also be obtained via a simpler and more direct approach, is of independent interest and it improves upon a previous solution approach recently proposed in [15], which required exponential enumeration. Robust linear programs with more general uncertainty structures are studied in [16].

\subsection{Polynomial-Time Solvable SDP Relaxation}

Although Theorem 2.1 entails a reduced set of vertex constraints, the number of such constraints still grows exponentially, and may happen to be too large for a practical solution. For these cases, we here propose a relaxation of the problem based on a sufficient LMI condition that guarantees the satisfaction of (2). 
Corollary 2.2 There exists $x \in \mathbb{R}^{m}$ that satisfies the robust interval LMI condition (2), if there exist $x \in \mathbb{R}^{m}$ and slack variables $\xi \in \mathbb{R}^{m}, t \in \mathbb{R}^{n}$ such that

$$
\begin{aligned}
& \bar{F}(x)+T \preceq 0, \\
& B(\xi) \preceq T, \\
& x_{k} \leq \xi_{k}, \quad k=1, \ldots, m, \\
& -x_{k} \leq \xi_{k}, \quad k=1, \ldots, m,
\end{aligned}
$$

where $T=\operatorname{diag}(t)$ and $\bar{F}(x), B(\xi)$ are defined in (5) and (10), respectively. An upper bound for the optimal value of Problem 2.2 is hence obtained by minimizing $c^{\top} x$ subject to the LMI constraints (12).

Proof If (12b) holds, then for any $S \in \mathcal{S}^{n}$ it holds that

$$
S B(\xi) S \preceq S T S \equiv T ;
$$

therefore, adding $\bar{F}(x)$ to both sides of this inequality we obtain that

$$
\bar{F}(x)+S B(\xi) S \preceq \bar{F}(x)+T .
$$

This inequality shows that (12a) implies (9b) in Theorem 2.1, thus concluding the proof.

\section{SPDs with Linearly Transformed Interval Uncertainty}

The main result treated in Sect. 2.2 refers to the situation when all entries of the LMI coefficient matrices are affected by independent interval uncertainty. In some specific applications, such as robust control, interval LMIs arise where the uncertainties are not independent. However, reduced vertex set results can be obtained also in these cases, as recently shown in [10].

Here we examine one of such non-independent uncertainty situations, where the LMI constraints are additively perturbed by a linear function of an interval matrix $\Delta$. Namely, we consider the uncertain LMI constraint

$$
F(x)=\bar{F}(x)+L \Delta R(x)+R^{\top}(x) \Delta^{\top} L^{\top} \preceq 0,
$$

where $\bar{F}(x)$ is an $n \times n$ symmetric affine matrix function of $x \in \mathbb{R}^{m}, R(x)$ is a $q \times n$ affine matrix function of $x, L \in \mathbb{R}^{n, p}$ is a given matrix, and $\Delta$ is a $p \times q$ interval matrix, i.e. $\Delta \in \mathcal{D}^{p, q}$, where

$$
\mathcal{D}^{p, q} \doteq\left\{\Delta \in \mathbb{R}^{p, q}:|\Delta| \leq B\right\},
$$

with $B \in \mathbb{R}^{p, q}$ a given nonnegative matrix representing the entrywise bounds on the absolute values of the elements of $\Delta$.

The uncertain LMI representation (13) is a special case of the classical linear fractional transformation (LFT; see, e.g., Sect. 2.2 of [6]), which frequently arises in robust control applications, see for instance $[17,18]$. The following theorem holds. 
Theorem 3.1 A vector $x \in \mathbb{R}^{m}$ satisfies the robust LMI condition

$$
\bar{F}(x)+L \Delta R(x)+R^{\top}(x) \Delta^{\top} L^{\top} \preceq 0, \quad \forall \Delta \in \mathcal{D}^{p, q},
$$

if and only if it satisfies

$$
\begin{gathered}
\bar{F}(x)+L S_{L} B S_{R} R(x)+R^{\top}(x) S_{R} B^{\top} S_{L} L^{\top} \preceq 0, \\
S_{L}=\operatorname{diag}\left(1, \tilde{S}_{L}\right), \quad \forall \tilde{S}_{L} \in \mathcal{S}^{p-1}, \forall S_{R} \in \mathcal{S}^{q} .
\end{gathered}
$$

Condition (15) consists of a finite number $2^{p+q-1}$ of vertex LMI constraints.

Proof The proof follows a reasoning similar to the one developed for the results in Sect. 2.2. Notice preliminarily that a vector $x \in \mathbb{R}^{m}$ satisfies (14) if and only if

$$
v^{\top} \bar{F}(x) v+2 \max _{\Delta \in \mathcal{D}^{p, q}} v^{\top} L \Delta R(x) v \leq 0, \quad \forall v \in \mathbb{R}^{n} .
$$

Let $z^{\top} \doteq v^{\top} L \in \mathbb{R}^{1, p}, y(x) \doteq R(x) v \in \mathbb{R}^{q, 1} ;$ then,

$$
\max _{\Delta \in \mathcal{D}^{p, q}} v^{\top} L \Delta R(x) v=\max _{\Delta \in \mathcal{D}^{p, q}} z^{\top} \Delta y(x)=\max _{\Delta \in \mathcal{D}^{p, q}} \sum_{i=1}^{p} \sum_{j=1}^{q} \Delta_{i j} z_{i} y_{j}(x)
$$

[since the max is attained for $\Delta_{i j}=B_{i j} \operatorname{sign}\left(z_{i} y_{j}(x)\right)$ ]

$$
\begin{aligned}
& =\sum_{i=1}^{p} \sum_{j=1}^{q} B_{i j}\left|z_{i} y_{j}(x)\right|=\sum_{i=1}^{p} \sum_{j=1}^{q}\left|z_{i}\right| B_{i j}\left|y_{j}(x)\right| \\
& =|z|^{\top} B|y(x)|=\left|v^{\top} L\right| B|R(x) v| .
\end{aligned}
$$

From (16), we thus have the following preliminary result: (14) holds if and only if

$$
v^{\top} \bar{F}(x) v+\left|v^{\top} L\right| B|R(x) v|+|R(x) v|^{\top} B^{\top}\left|v^{\top} L\right|^{\top} \leq 0, \quad \forall v \in \mathbb{R}^{n} .
$$

Now, suppose first that (14) holds. Then, for any $S_{L} \in \mathcal{S}^{p}$ and any $S_{R} \in \mathcal{S}^{q}$, since $\Delta=S_{L} B S_{R} \in \mathcal{D}^{p, q}$, we see immediately that (15) is satisfied.

Conversely, suppose (15) holds, and notice that whenever (15) is satisfied for a pair $\left(S_{L}, S_{R}\right)$ it is also satisfied for the pair $\left(-S_{L},-S_{R}\right)$. This implies that, despite the restriction on the sign of the first diagonal element in $S_{L},(15)$ actually holds for all $S_{L} \in \mathcal{S}^{p}$ and for all $S_{R} \in \mathcal{S}^{q}$. Therefore, for all $v \in \mathbb{R}^{n}$, it holds that

$$
\begin{aligned}
& v^{\top} \bar{F}(x) v+v^{\top} L S_{L} B S_{R} R(x) v+v^{\top} R^{\top}(x) S_{R} B^{\top} S_{L} L^{\top} v \leq 0, \\
& \forall S_{L} \in \mathcal{S}^{p}, \forall S_{R} \in \mathcal{S}^{q} .
\end{aligned}
$$

In particular, by choosing

$$
\begin{aligned}
& S_{L}=\operatorname{diag}\left(\operatorname{sign}\left(\left[v^{\top} L\right]_{1}\right), \ldots, \operatorname{sign}\left(\left[v^{\top} L\right]_{p}\right)\right), \\
& S_{R}=\operatorname{diag}\left(\operatorname{sign}\left([R(x) v]_{1}\right), \ldots, \operatorname{sign}\left([R(x) v]_{q}\right)\right),
\end{aligned}
$$


we have that, for all $v \in \mathbb{R}^{n}$, it must hold that

$$
v^{\top} \bar{F}(x) v+\left|v^{\top} L\right| B|R(x) v|+|R(x) v|^{\top} B^{\top}\left|v^{\top} L\right|^{\top} \leq 0,
$$

which is (17). Since we proved in the preliminary result that (17) is equivalent to (14), we concluded our proof.

Remark 3.1 Theorem 3.1 and its proof are closely related to the main result of [10]. In particular, the authors of [10] consider a problem arising in a robust control setting which, restated in the notation of this paper, takes the following form:

$$
\bar{F}(x)+\Delta_{a}+\Delta_{a}^{\top}+\Delta_{b} Q(x)+Q^{\top}(x) \Delta_{b} \preceq 0, \quad \forall \Delta_{a} \in \mathcal{D}^{n, n}, \Delta_{b} \in \mathcal{D}^{n, m} .
$$

It is proved in [10] that this condition is equivalent to $2^{m+n}$ conditions on specific vertex matrices. We next show that (18) is a special case of LMI (14), and that Theorem 3.1 can be modified and specialized to this case, thus providing a vertex cardinality result that improves by an halving factor the $2^{m+n}$ vertex set cardinality result of [10]. To this end, notice that (18) can be written in the form (14), by taking

$$
\Delta=\left[\begin{array}{ll}
\Delta_{a} & \Delta_{b}
\end{array}\right] \in \mathbb{R}^{n, n+m}, \quad L=I_{n}, \quad R^{\top}(x)=\left[\begin{array}{ll}
I_{n} & Q^{\top}(x)
\end{array}\right] .
$$

Then, it can be easily verified that all steps in the proof of Theorem 3.1 would go through with $S_{L}=\operatorname{diag}\left(1, \tilde{S}_{L}\right), \tilde{S}_{L} \in \mathcal{S}^{n-1}$, and with $S_{R}$ taking the specific block structure $S_{R}=\operatorname{diag}\left(S_{L}, S_{Q}\right)$, with $S_{Q} \in \mathcal{S}^{m}$. The resulting condition of type (15) would thus involve only $2^{m+n-1}$ vertex constraints,

$$
\bar{F}(x)+S_{L} B_{a} S_{L}+S_{L} B_{b} S_{Q} Q(x) \preceq 0, \quad S_{L}=\operatorname{diag}\left(1, \tilde{S}_{L}\right), \forall \tilde{S}_{L} \in \mathcal{S}^{n-1}, S_{Q} \in \mathcal{S}^{m}
$$

where $\left[\begin{array}{ll}B_{a} & B_{b}\end{array}\right]$ is the matrix of bounds for the interval matrix $\Delta=\left[\begin{array}{ll}\Delta_{a} & \Delta_{b}\end{array}\right]$.

Finally, we provide a result which is the analog of Theorem 3 in [10]. This result gives an efficiently computable sufficient condition for the satisfaction of (14) and it is reported in Corollary 3.1 with an alternative proof.

Corollary 3.1 If $x \in \mathbb{R}^{m}, \Theta=\operatorname{diag}\left(\theta_{1}, \ldots, \theta_{q}\right) \succ 0, T=\operatorname{diag}\left(t_{1}, \ldots, t_{p}\right)$ satisfy the LMIs

$$
\begin{aligned}
& {\left[\begin{array}{cc}
\bar{F}(x)+L T L^{\top} & R^{\top}(x) \\
R(x) & -\Theta
\end{array}\right] \preceq 0,} \\
& B \Theta B^{\top} \preceq T
\end{aligned}
$$

then $x$ satisfies (14).

Proof Let $\overline{\mathcal{S}}^{q} \doteq\left\{\operatorname{diag}\left(s_{1}, \ldots, s_{q}\right):\left|s_{i}\right| \leq 1, i=1, \ldots, q\right\}$. Obviously, we have that $\mathcal{S}^{q} \subset \overline{\mathcal{S}}^{q}$; hence,

$$
\bar{F}(x)+L S_{L} B S_{R} R(x)+R^{\top}(x) S_{R} B^{\top} S_{L} L^{\top} \preceq 0, \quad \forall S_{L} \in \mathcal{S}^{p}, \forall S_{R} \in \overline{\mathcal{S}}^{q},
$$


implies that (15) is satisfied. In turn, applying the relaxation result in Lemma 3.2 of [6], we have that (20) is satisfied if there exists a diagonal matrix of positive scalings $\Theta=\operatorname{diag}\left(\theta_{1}, \ldots, \theta_{q}\right) \succ 0$ such that

$$
\left[\begin{array}{cc}
\bar{F}(x)+L S_{L} B \Theta B^{\top} S_{L} L^{\top} & R^{\top}(x) \\
R^{\top}(x) & -\Theta
\end{array}\right] \preceq 0, \quad \forall S_{L} \in \mathcal{S}^{p} .
$$

Now let $T=\operatorname{diag}\left(t_{1}, \ldots, t_{p}\right)$ be such that $B \Theta B^{\top} \preceq T$. This implies that, for any $S_{L} \in$ $\mathcal{S}^{p}, S_{L} B \Theta B^{\top} S_{L} \preceq S_{L} T S_{L} \equiv T$, and, by congruence, $L S_{L} B \Theta B^{\top} S_{L} L^{\top} \preceq L T L^{\top}$, for all $S_{L} \in \mathcal{S}^{p}$. Substituting the second term in the sum in the $(1,1)$ block of the LMI (21) with the majorizing term $L T L^{\top}$, we prove that (19) imply (15). The claim hence follows by application of Theorem 3.1.

\section{Numerical Example}

We revisit a problem originally considered in [19], dealing with the minimization of the largest eigenvalue of an affine combination of symmetric matrices. Namely, in [19], the following problem is considered:

$$
\min _{x \in \mathbb{R}^{5}} \lambda_{\max }\left(\bar{A}_{0}+\sum_{i=1}^{5} x_{i} \bar{A}_{i}\right)
$$

where $\lambda_{\max }$ denotes the largest eigenvalue of a symmetric matrix and $\bar{A}_{0}, \ldots, \bar{A}_{5}$ are the following symmetric matrices:

$$
\begin{aligned}
\bar{A}_{0} & =\left[\begin{array}{rrrrr}
-0.69 & -0.32 & 0.34 & 0.43 & -0.05 \\
-0.32 & -0.11 & -0.11 & -0.45 & -0.34 \\
0.34 & -0.11 & -0.71 & -0.33 & -0.08 \\
0.43 & -0.45 & -0.33 & 0.65 & 0.27 \\
-0.05 & -0.34 & -0.08 & 0.27 & 0.39
\end{array}\right], \\
\bar{A}_{1} & =\left[\begin{array}{rrrrr}
-0.66 & 0.31 & 0.57 & -0.06 & -0.44 \\
0.31 & -0.23 & -0.12 & -0.35 & 0.28 \\
0.57 & -0.12 & -0.26 & -0.06 & -0.37 \\
-0.06 & -0.35 & -0.06 & 0.64 & 0.34 \\
-0.44 & 0.28 & -0.37 & 0.34 & 0.61
\end{array}\right], \\
\bar{A}_{2}= & {\left[\begin{array}{rrrrr}
-0.31 & 0.35 & 0.06 & -0.23 & 0.17 \\
0.35 & 0.24 & -0.19 & 0.21 & -0.12 \\
0.06 & -0.19 & -0.34 & 0.00 & -0.36 \\
-0.23 & 0.21 & 0.00 & 0.16 & -0.24 \\
0.17 & -0.12 & -0.36 & -0.24 & 0.00
\end{array}\right], }
\end{aligned}
$$




$$
\begin{aligned}
\bar{A}_{3} & =\left[\begin{array}{rrrrr}
0.27 & -0.14 & 0.13 & -0.32 & -0.08 \\
-0.14 & -0.20 & -0.29 & -0.05 & -0.64 \\
0.13 & -0.29 & -0.45 & -0.20 & -0.59 \\
-0.32 & -0.05 & -0.20 & -0.27 & -0.46 \\
-0.08 & -0.64 & -0.59 & -0.46 & -0.39
\end{array}\right], \\
\bar{A}_{4} & =\left[\begin{array}{rrrrr}
-0.57 & -0.38 & -0.09 & 0.31 & 0.22 \\
-0.38 & 0.66 & 0.17 & -0.03 & 0.51 \\
-0.09 & 0.17 & 0.23 & 0.12 & -0.21 \\
0.31 & -0.03 & 0.12 & -0.56 & -0.21 \\
0.22 & 0.51 & -0.21 & -0.21 & 0.59
\end{array}\right], \\
\bar{A}_{5} & =\left[\begin{array}{rrrrr}
0.22 & 0.28 & 0.14 & 0.03 & 0.09 \\
0.28 & 0.69 & -0.12 & 0.10 & 0.30 \\
0.14 & -0.12 & -0.77 & -0.21 & 0.13 \\
0.03 & 0.10 & -0.21 & -0.42 & -0.15 \\
0.09 & 0.30 & 0.13 & -0.15 & 0.22
\end{array}\right] .
\end{aligned}
$$

Problem (22) can be recast in SDP form as

$$
\begin{array}{ll}
\min _{x \in \mathbb{R}^{5}, \lambda \in \mathbb{R}} & \lambda, \\
\text { s.t. } & \bar{A}_{0}+\sum_{i=1}^{5} x_{i} \bar{A}_{i}-\lambda I \preceq 0,
\end{array}
$$

which has the optimal solution $\lambda_{\min }=0.70888$.

Assume now that the matrices $\bar{A}_{1}, \ldots, \bar{A}_{5}$ represent nominal values, while the actual entries are only known to lie in independent intervals centered around the nominal values, with width equal to $\rho$ percent of the nominal, that is,

$$
A_{k}=\bar{A}_{k}+\Delta_{k}, \quad\left|\Delta_{k}\right| \leq \rho\left|\bar{A}_{k}\right| ; k=1, \ldots, 5 .
$$

In this situation, the problem becomes that of minimizing the worst-case largest eigenvalue of the interval matrix family, that is,

$$
\begin{array}{ll}
\min _{x \in \mathbb{R}^{5}, \lambda \in \mathbb{R}} & \lambda, \\
\text { s.t. } & \bar{A}_{0}+\sum_{i=1}^{5} x_{i} \bar{A}_{i}+\sum_{i=1}^{5} x_{i} \Delta_{i}-\lambda I \preceq 0, \\
& \forall\left|\Delta_{k}\right| \leq \rho\left|\bar{A}_{k}\right|, k=1, \ldots, 5 .
\end{array}
$$

This problem is an interval SDP of the form (2).

Determining a robust solution for this interval SDP using a naive vertex approach would require considering $2^{50} \approx 10^{15}$ vertices. Application of Theorem 2.1 requires instead only 16 vertices. Hence, solving this problem for increasing values of $\rho$ ranging in the interval $[0,1]$ (which corresponds to uncertainty level from $0 \%$ to $100 \%$ ), we obtained the plot shown in Fig. 1. This numerical example was coded in Matlab using the YALMIP interface (see [20]) and the SeDuMi SDP solver. The numerical 
Fig. 1 Plot of the worst-case largest eigenvalue $\lambda$ max as a function of $\rho$

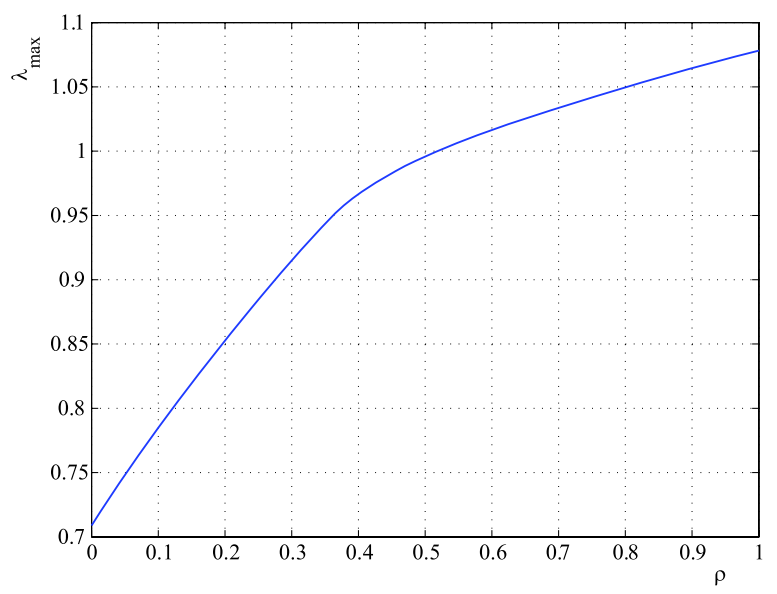

solution of the problem for each fixed value of $\rho$ required about 0.13 seconds on an AMD Dual Opteron workstation.

\section{Conclusions}

Robust solutions to uncertain SDP problems with interval coefficient matrices require considering an exponential number of vertex constraints. We have shown in this paper that when all entries of the LMI coefficient matrices lie in independent intervals, the number of vertices to be considered in the optimization is $2^{n-1}$, being $n$ the size of the LMI. Interval linear programs are a special case of the considered class of problems, and can be solved efficiently without resorting to vertexization. When the LMI constraint is expressed as a linear transformation of a $p \times q$ matrix of uncertain coefficients, the required number of vertices becomes $2^{p+q-1}$.

\section{References}

1. Boyd, S., El Ghaoui, L., Feron, E., Balakrishnan, V.: Linear Matrix Inequalities in System and Control Theory. SIAM, Philadelphia (1994)

2. Nesterov, Y., Nemirovski, A.: Interior-Point Polynomial Algorithms in Convex Programming. SIAM, Philadelphia (1995)

3. Todd, M.: Semidefinite optimization. Acta Numer. 10, 515-560 (2001)

4. Vandenberghe, L., Boyd, S.: Semidefinite programming. SIAM Rev. 38, 49-95 (1996)

5. Ben-Tal, A., Nemirovski, A.: Robust convex optimization. Math. Oper. Res. 23, 769-805 (1998)

6. El Ghaoui, L., Oustry, F., Lebret, H.: Robust solutions to uncertain semidefinite programs. SIAM J. Optim. 9, 33-52 (1998)

7. Blondel, V., Tsitsiklis, J.: A survey of computational complexity results in systems and control. Automatica 36, 1249-1274 (2000)

8. Nemirovski, A.: Several NP-hard problems arising in robust stability analysis. Math. Control Signals Syst. 6, 99-195 (1993)

9. Ben-Tal, A., Nemirovski, A.: On tractable approximations of uncertain linear matrix inequalities affected by interval uncertainty. SIAM J. Optim. 12(3), 811-833 (2002)

10. Alamo, T., Tempo, R., Ramirez, D., Camacho, E.: A new vertex result for robustness problems with interval matrix uncertainty. Syst. Control Lett. 57, 474-481 (2008) 
11. Rohn, J.: Positive definiteness and stability of interval matrices. SIAM J. Matrix Anal. Appl. 15, 175-184 (1994)

12. Rohn, J.: Checking positive definiteness or stability of symmetric interval matrices is NP-hard. Comment. Math. Univ. Carol. 35(4), 785-797 (1994)

13. Ben-Tal, A., Nemirovski, A.: Robust truss topology design via semidefinite programming. SIAM J. Optim. 7(4), 991-1016 (1997)

14. Vandenberghe, L., Boyd, S.: Applications of semidefinite programming. Appl. Numer. Math. 29(3), 283-299 (1999)

15. Chinneck, J., Ramadan, K.: Linear programming with interval coefficients. J. Oper. Res. Soc. 51, 209-220 (2000)

16. Ben-Tal, A., Nemirovski, A.: Robust solutions of uncertain linear programs. Oper. Res. Lett. 25(1), 1-13 (1999)

17. Petersen, I.: A stabilization algorithm for a class of uncertain systems. Syst. Control Lett. 8, 351-357 (1987)

18. Zhou, K., Doyle, J., Glover, K.: Robust and Optimal Control. Prentice-Hall, Upper Saddle River (1996)

19. Fan, M., Nekooie, B.: On minimizing the largest eigenvalue of a symmetric matrix. In: Proceedings of the IEEE Conference on Decision and Control (1992)

20. Lofberg, J.: YALMIP: A toolbox for modeling and optimization in MATLAB. In: Proceedings of the CACSD Conference, Taipei, Taiwan (2004) 